lation, first the general theory and then that for four-fold tables. Chapter XIII is a tantalizing chapter. It begins with the statement that two phenomena are correlated, when in whole or in part they are the resultants of the same elementary causes, and then simply gives the ordinary productmoment formula for the correlation coefficient with plans for calculating the various constants.

Interested students will find Charlier's methods discussed in considerable detail in Arne Fisher's recent work, The Mathematical Theory of Probability.

\title{
A. R. Crathorne
}

Théorie Mathématique des Phénomènes Thermiques produits par la Radiation Solaire. By M. Milankovitch. Paris, Gauthier-Villars, 1920. $\mathrm{xvi}+340 \mathrm{pp}$.

This book is of great interest in several respects. It is written by a Serb and was interrupted by war when the author was taken prisoner by Austria-Hungary. Granted the freedom to pursue his work the book was completed owing to the courtesy of the Hungarian Academy of Sciences. But its greater title to interest is that, apparently, it is the first complete treatise on the thermal effects of solar radiation treated from a systematic point of view.

At the first stage the problem is considered of the amount of heat received per unit area on a rotating planet devoid of atmosphere with a sun fixed in distance and direction. Account is then taken of the elliptic orbit and obliquity of the ecliptic to determine the "radiation-constant" day by day. In particular, the amount of heat received in each season of the year is expressed by formula.

Up to this point the work is practically a branch of celestial kinematics. At the next step the loss of radiation due to absorption by an atmosphere etc. is considered, especially as it tends to diminish the intensity of oblique rays. This factor has a large influence on the temperature of points near the arctic circle. It is due to this factor that a variation in the obliquity of the ecliptic may produce large changes in the climate of higher latitudes.

Passing over an excursion relative to the effect of conduction of heat in the solid crust, the next topic is the determination of the loss caused by the atmosphere. This loss consists partly of scattering in the air, and reflection by clouds and by the surface of the earth, partly by true absorption with re-emission at a much longer wave-length. One outstanding fact is that the atmosphere absorbs far more of the outwardly emitted long-wave radiation than of the incoming visible light.

To give a mathematical theory it is necessary to consider the thermal mechanics of gases. Two simplifying assumptions may be made. Of these, adiabatic equilibrium is shown to lead to results quite out of keeping with observation. On the other hand, the assumption of temperature equilibrium under absorption and emission of radiation appears to fit the facts well, and when there are two gases with distinct properties a reversal of temperature gradient is shown theoretically to be possible. A particular example is given by a mixture of oxygen and carbon dioxide such as that actually in the atmosphere. 
Finally the theory is applied to the special case of the earth and, also, to the three other minor planets, and for the earth numerical tables are given. The question of glacial periods is handled in a moderate spirit, critically and without controversial prejudice. Since glaciation is not caused by cold winters (cf. Siberia) but by long and cold summers (that is, by cool periods of precipitation) the effect on temperate climates of a variation in the obliquity of the ecliptic combined with a high eccentricity of orbit may be quite large.

Stockwell's formula for the variations of $e$ and $\epsilon$ are used to calculate the amount of heat received each season and the length of each season for the 500 millennia before 1850 . This formula is of so complicated and empirical a form that one can have little confidence in the extreme degree of extrapolation which such a use implies. It would seem to be enough, however, to warrant the conclusion that glacial epochs could be explained if the variations have been in the past slightly greater than those given by the exact formula.

The author is to be warmly congratulated on such a thorough and critical attack on the problem of "mathematical climate." The book can be recom mended, not only to meteorologists, but also to those who enjoy the clearing up of new fields in physics by the use of straightforward mathematics with numerical determinations. It would exert a broadening influence on a student of mathematical physics in whose hands it might be placed, since it combines problems from several distinct fields.

\section{P. J. Danieli}

Géométrie Descriptive. By Gaspard Monge. Augmentée d'une Théorie des Ombres et de la Perspective, extraite des papiers de l'auteur par Barnabé Brisson. Parts I and II. Paris, Gauthier-Villars, 1922. xvi + $144+138$ pp.

These reprints, appearing in the series Les MaItres de LA Penstee ScIentifique ably edited by Maurice Solovine, are of special interest to Americans for the reason that the descriptive geometry created by Monge was among the earliest mathematical creations of the French which reached the United States; it was taught at West Point in the first quarter of the nineteenth century. Monge's descriptive geometry was first published in the year VII (1799), but the present reprint is from the fourth (1820) posthumous edition which included also material on shadows and perspective, selected by Brisson from Monge's then unpublished papers. Of interest is Monge's estimate of the value of descriptive geometry: "If in all the larger towns there were established secondary schools, in which young men of the age of twelve years who look forward to practicing some one of the trades should be trained during two years in graphic constructions and become acquainted with the principal phenomena of nature, a knowledge of which is indispensable to them, this training, in developing their intelligence and giving them the habit and the sentiment of precision, would contribute in the surest manner to the progress of national industry." 